\title{
Design and Molecular Docking of Antioxidant Lead Compound and its Analogues Acting as Human Tyrosine Kinase Inhibitors
}

\author{
Ammara Khalid $^{1}$, SaimaKalsoom ${ }^{2}$, NaveedaRiaz ${ }^{* 1}$ \\ ${ }^{1}$ (Department of Microbiology and Molecular Genetics, University of the Punjab, Pakistan) \\ ${ }^{I}$ (Department of Bioinformatics and Biotechnology, International Islamic University, Islamabad, Pakistan) \\ 2 (Department of Chemistry, Quaid-i-Azam University, Islamabad, Pakistan)
}

\begin{abstract}
Cancer is the $2^{\text {nd }}$ leading cause of death in humans. Proteine.g. tyrosine kinaseleads to cancer when become constitutively active due to mutation. So there is a need to develop tyrosine kinase inhibitors to avoid cancer.In the present study the problem of designing an anticancer drug with more efficacies was solved by using computer aided drug designing (CADD). Antioxidants were used as tyrosine kinase inhibitors. Molecular docking studies of antioxidants belonging from different classes were carried out with human target protein having pdbid:2HCK in order to find out the most active antioxidant drug having high inhibitory activity in cancer.Docking of 36 selected ligands, comprised of active drugs for cancer and compounds having anticancer activity, was done with the active site of protein $2 \mathrm{HCK}$ and a lead compound was recognized on the basis of hydrogen bonding, hydrophobic and ionic interactions. Three analogues of the lead compound were designed to enhance its activity against cancer. Analogues were docked with protein $2 \mathrm{HCK}$ and their interactions showed that they could possess good inhibitory activity against cancer with suitable drug-like properties as compared to other active drugs for cancer and therefore could be recommended for further studies.
\end{abstract}

Keywords: Antioxidants, Tyrosine Kinase inhibitors, lead compound, Cancer, Molecular docking

\section{Introduction}

Antioxidants are the bioactive chemical compounds which are highly important to fight against oxidative stress which is responsible for various diseases including cancer. Oxidative stress produces due to the accumulation of free radicals. Free radicals interfere with different process in the body by destroying proteins, lipids and DNA sugars etc[1]. Antioxidant fight against oxidative stress by neutralizing the effect of free radicals produce during the process of respiration as a by-product of oxygen metabolism and thus can play important role in preventing many life threatening diseases. Antioxidant helps in neutralizing the free radicals before they do harm to our body antioxidant system includes different enzymes such as superoxide dismutase, glutathione reductase, glutathioneperoxidase, S-methyl transferase and catalase. Small molecule antioxidants such as ascorbate, glutathione, tocopherol, carotenoids, polyphenols, alkaloids and some other compounds are also important cellular antioxidants [2].

Tyrosine kinases are a family of proteins involved in the transduction of growth factor signals to the nucleus and are located in or near the cell membrane.Tyrosine kinases catalyze the phosphorylation of tyrosine residues in proteins. The phosphorylation of tyrosine residues then causes a change in the function of the protein in which they are present in. Due to mutations some tyrosine kinases become constitutively active and nonstop functional state which can result in cancer[3].

Querecetin is an antioxidant compound also used as tyrosine kinase inhibitor. But quercetin appears to be associated with side effects when administered orally and intravenously[4]for example, it may cause kidney failure, gastrointestinal complaints, allergic skin reactions, and increase the risk of kernicterus (brain damage due to severe jaundice) in newborns, mouth sores, headaches, and digestive upset are also the possible side effects of taking quercetin[5]. There is a need to develop more antioxidant drugs which are more specific and significantly fewer side effects, can bind with tyrosine kinase, and inhibits its hyper activity which is one of the reasons for cancer formation.

The studies have been carried out in order to identify effective, selective and efficient anticancerous antioxidant Lead compound and its analogues. In short, this work reveals that the current bioinformatics tools used strategically can lead to the discovery of potential Lead compounds and their analogues that can inhibit the process of cancer formation. In current study molecular docking has been carried out on different antioxidants with the aim of identifying their anticancer activity in Human tyrosine kinase binding and inhibition. ChemDraw [6], AutodockVina[7] and visual molecular dynamics (VMD) [8] were used for studying molecular docking and ligand-protein interactions, respectively. 


\subsection{Target selection}

\section{Methodology}

Identifying and selecting the most appropriate drug target or receptor is the key step in drug designing.Bioinformatics tools can be used to identify the required target. For a drug target to be ideal it must be specifically linked to the human disease. Inpresent study protein having pdb id: $2 \mathrm{hck}$ shown in Fig. 1 is used.

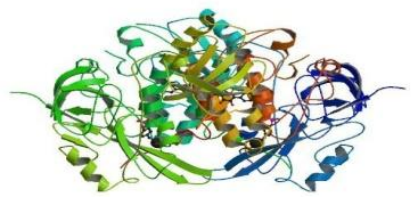

Figure 1.Crystal structure of hematopoietic cell kinase (HCK) quercetin complex.

\subsection{Ligand selection}

Various antioxidant tyrosine kinase inhibitors have been identified and selected in this study. The data set consisted of 36 antioxidant ligands(A1-A36) have drug like properties against cancer.Ligands were drawn by using ChemDraw. All the ligands were selected on the bases of clearance from 2,2-Diphenyl-1-Picrylhydrazyl (DPPH) bioassayand the IC50 of all the ligands should lie under $100 \mu \mathrm{M}$. Structures and IC50 values of these 36 compounds along with the standard compound Quercetin (A11) are shown in Table 1 [1, 2, 9-14].

Table 1. Chemical structures and IC50 values of selected compounds

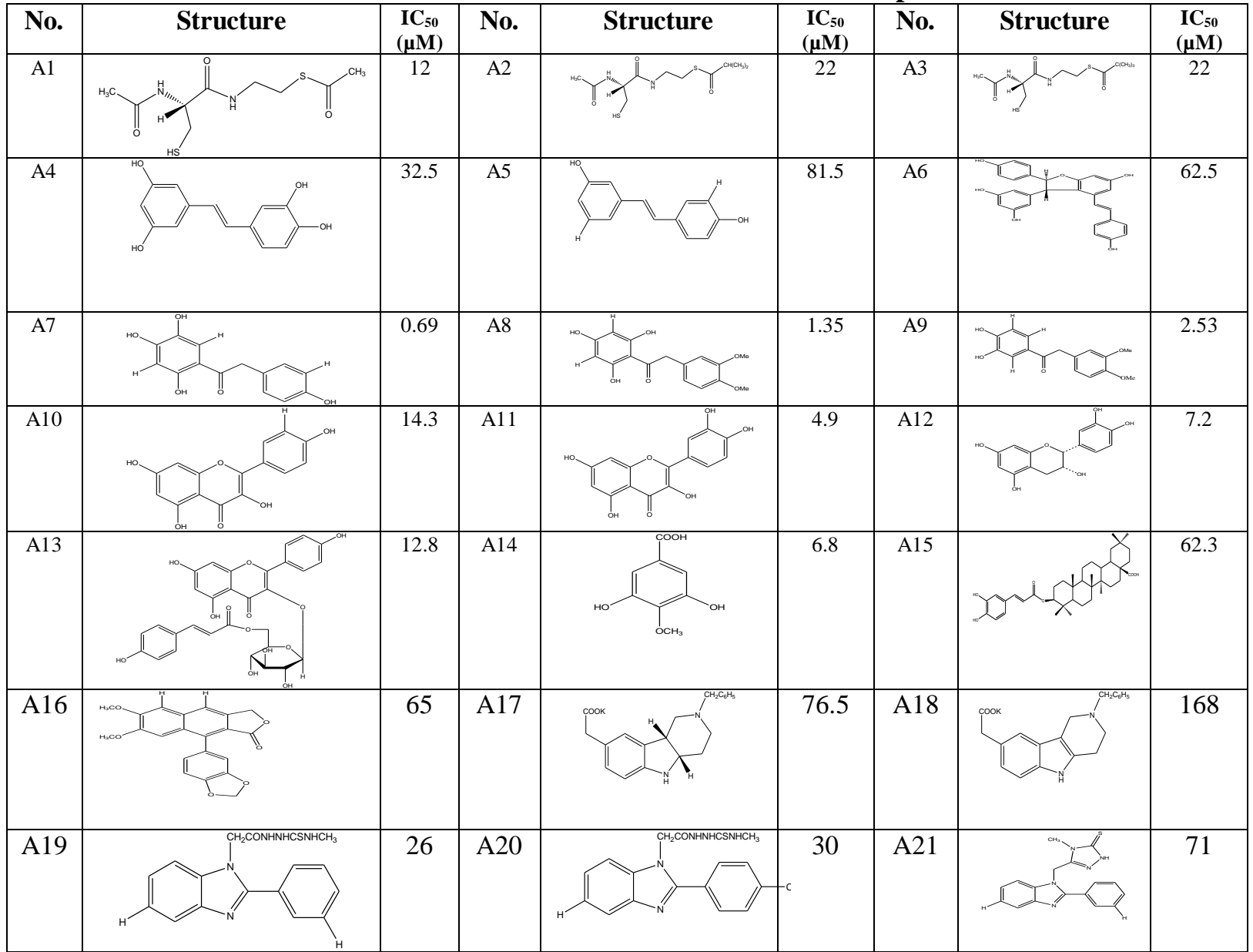




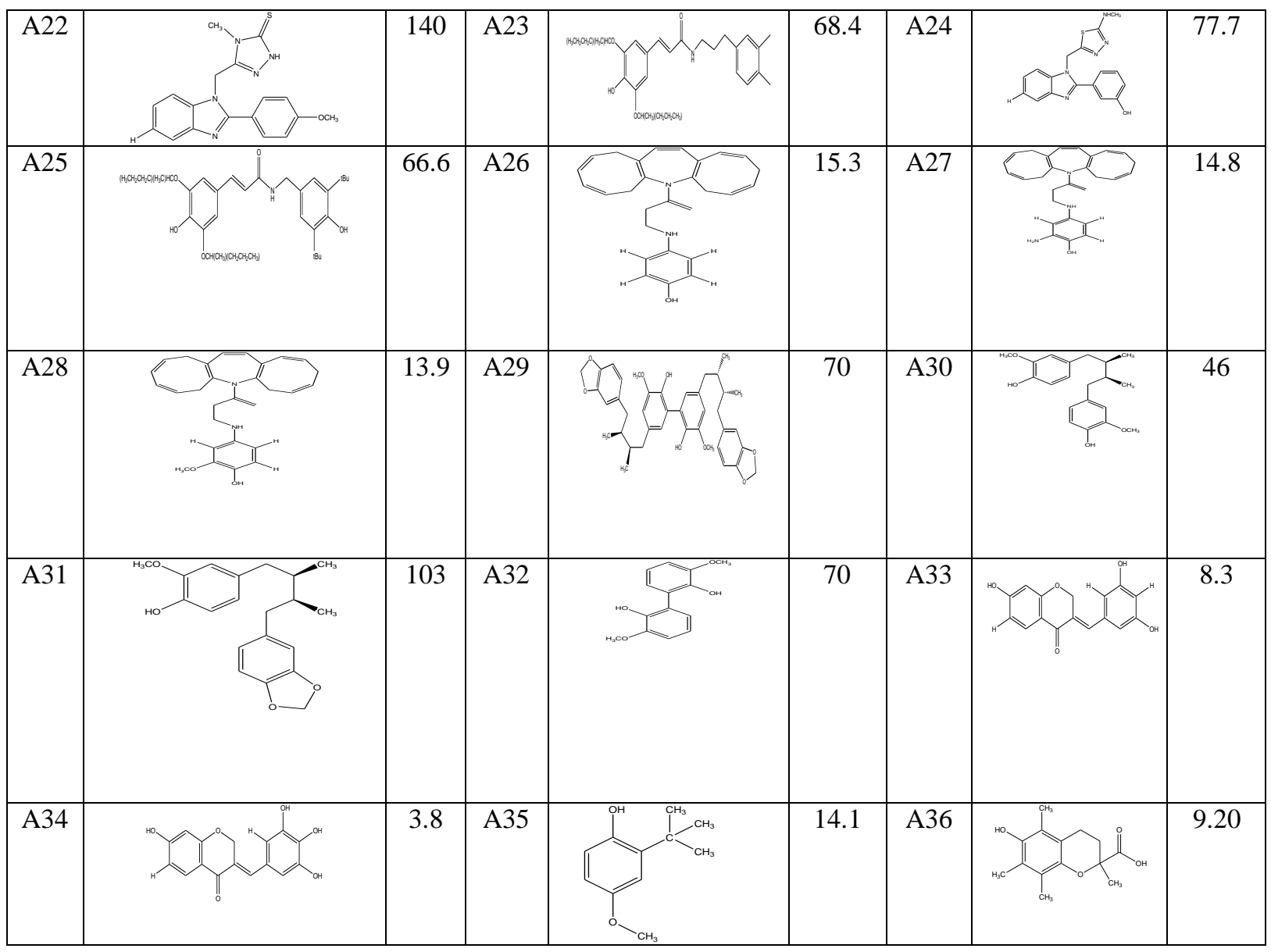

\subsection{Molecular docking}

In molecular docking the ligand and the target protein are two important ingredients. In order to perform docking a specific target protein was chosen for antioxidant compounds. Tyrosine kinase (pdb id: $2 \mathrm{HCK}$ ) was identified as protein target for current studies. Pdb file $2 \mathrm{HCK}$ was obtained from the protein data bank (rscb.org).AutodockVina was used for docking in this study. AutodockVina is the latest version of Autodock it provides speed and accuracy and it accepts the ligand and the target protein in pdb format. The pdb files of the macromolecule $2 \mathrm{HCK}$ and ligand were placed in a newly made folder. The pdb file of the macromolecule (protein) was given as an input to the software. Protein was colored after that polar hydrogen's were added to it. This macromolecule was then saved as grid output file with .pdbqt extension. Then in grid box different values were set according to the active site of protein. After this ligand pdb file was taken as an input and its torsions were calculated this ligand file was also saved with .pdbqt extension. Another file of .txt extension was made in which receptor and ligand names and there location of binding at $\mathrm{x} y$ and $\mathrm{z}$ axis with each other were set. The.txt file was used in command prompt as an input file and command prompt itself generates an output log file with .txt extension according to the given input file. After generating log.txt file different docking conformations were obtained. The conformation having lowest energy of all the compounds was chosen to find out the interactions with target protein. This previously mentioned procedure of docking was done on all compounds.

The ligand-protein interactions were predicted using Visual Molecular Dynamics (VMD). The most active antioxidant tyrosine kinase inhibitor was identified after the detailed analysis of ligand-protein interactions. Binding interactions of all compounds were observed and then the compound showing best interactions among all was selected as Lead. Lead designing is the most crucial step in drug designing and discovery.

\subsection{Analogue designing}

After the identification of Lead compound three structural analogues of the Lead were made by introduction or elimination of various functional groups in the Lead compound. Docking was performed on these analogues and their interactions with protein were identified. 


\subsection{Molecular docking}

\section{Results}

The binding interactions of the actively docked conformations of the ligands and the target protein have been identified and marked using VMD. Checking one by one all amino acids within $5 \mathrm{~A}^{\circ}$ of the active site of the target protein, the important binding interactions were identified. The identified interactions in all 36 compounds including the standard one include the hydrophobic interactions and hydrogen bonding. The binding interactions of all ligands have been analyzed. Five compounds have been identified as the most active from the set of given ligands A7, A12, A15, A25 and A33. These ligands have shown strong hydrogen bonding and hydrophobic interactions with the target protein than the rest of the ligands. Along with their strong interactions, the IC50 values of them are lower which is the positive sign towards their being the active ones. These three active ligands along with their interactions and IC50 values are shown in Table 2. From the table it is clear that the number of hydrogen bonds in ligands A7, A12, A15, A25 and A32 are 8,4,6,5 and 4 respectively, and the hydrophobic interactions are 2,1,2,2 and 2 respectively and additionally compounds A7 and A12 both showed 2 Vander Waal interactions as well. The lead compound has been identified from the 5 most active compounds, after analyzing them on the basis of their interactions and IC50 values. Among them ligand A7 has been identified as the lead compound. The strong ligand-protein interactions, which include 8hydrogen bonds and 2 hydrophobic interactions and 2 Vander Waal interactions is shown in Fig. 2 and the low IC50 amongst the five proved it to be the most active one. The interactions shown in Fig. 2, highlights the important amino acids of the target protein pocket and the atoms of the ligand. Fig. 2 also shows the bonding distances of the ligand with target protein. Compound A7 consisted of 8 hydrogen bonds i.e. hydrogen bond with amino acid SER247 with a distance of 3.56 amino acids TYR136, LYS151, VAL137, TYR90, GLU147, TYR90 and VAL137 with the distances of $3.41,2.88,3.27,2.76,3.84,3.94$ and $3.15 \mathrm{~A}^{0}$ respectively, A7 consisted of two hydrophobic interactions with amino acid LEU89 and GLU144 with the distance of 3.64 and $3.85 \mathrm{~A}^{0}$ respectively and two hydrophobic interactions with amino acids PHE150 and TYR90 with the distance of 3.52 and $3.78 \mathrm{~A}^{0}$ and respectively.

The interactions of the standard compound have also been identified and are shown in Fig. 3. The binding interactions of Quercetin include 4 hydrogen bonds, 2 hydrophobic interactions and 1 Vander Waal interaction. The hydrogen bonds include O GLU117 at a distance of 2.96, N of GLU117 at a distance of 2.97, O of GLN318 at a distance of 3.72 and $\mathrm{N}$ of GLN318 at a distance of 3.13with the standard compound. Hydrophobic interactions include C of LYS257 at a distance of 3.96 and C of HIS326 at a distance of 3.68 with standard compound. Vander Waal interaction is between hydrophobic rings of ligand with hydrophobic ring containing amino acid TRP254 of active site at a distance of 3.42 .

The binding interactions of the standard Quercetin A11 are very much close to the identified lead compound infact the number of interactions showed by lead compound are more than the standard compound and the IC50 of A7 is also lower than the A11. This gives stronger evidence that the identified lead compound has high efficacy and has great potential to be a drug.

Table 2. Five active ligands

\begin{tabular}{|c|c|c|c|c|c|}
\hline Ligand & Structure & Hydrogen bonding & Hydrophobic interactions & Vander Waal & $\mathrm{IC}_{50}$ \\
\hline A7 & & $\begin{array}{lll}\text { SER247 } & \text { OH-O } & (3.56 \mathrm{As} \\
\text { TYR136 } & \text { No } & (3.41 \\
\mathrm{As}\end{array}$ & LEU89 C.C (3.64 $\left.\mathrm{D}^{\circ}\right)$ & $\begin{array}{l}\text { PHE150 } \mathrm{CC}\left(3.52 \mathrm{~A}^{\circ}\right) \\
\text { TYR90 C.C }\left(3.78 \mathrm{~A}^{\circ}\right)\end{array}$ & 0.69 \\
\hline A12 & & 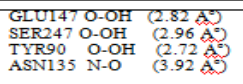 & LEUS9 C.C (3.71 $2 \mathrm{~g}^{\circ}$ & $\begin{array}{l}\text { PHE } 150 \mathrm{C}-\mathrm{C}(3.74 \mathrm{Ag} \\
\text { TYR } 90 \mathrm{C}-\mathrm{C}(3.93 \mathrm{Ag})\end{array}$ & 7.2 \\
\hline A15 & & 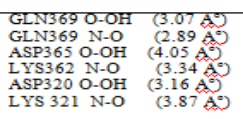 & 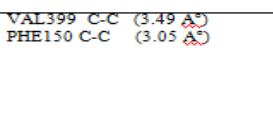 & & 62.3 \\
\hline A25 & & 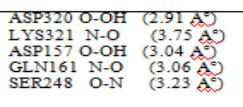 & 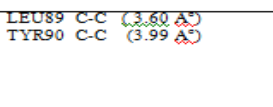 & & 66.6 \\
\hline A32 & & 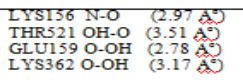 & 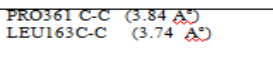 & & 70 \\
\hline
\end{tabular}




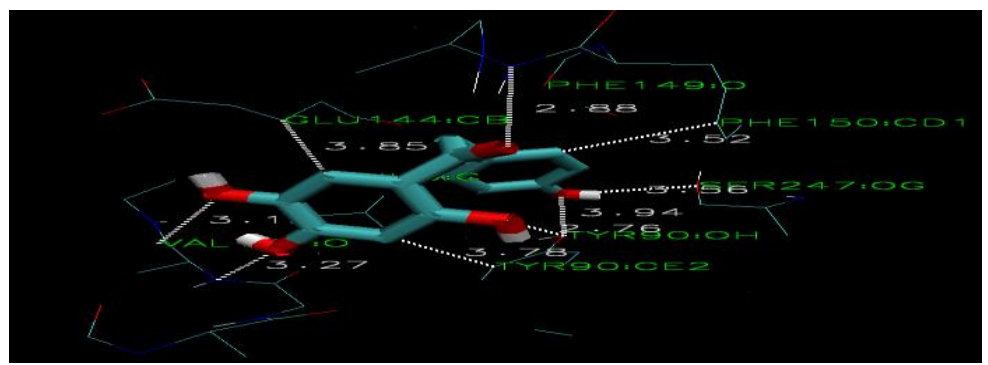

Figure 2. Binding interactions of Lead compound with target 2HCK

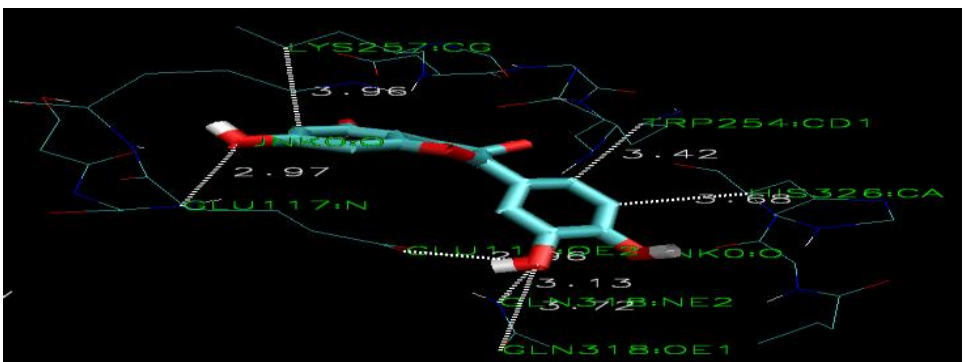

Figure 3. Binding interactions of Quercetin with target 2HCK

\subsection{Analogues of lead}

Three structural analogues of the lead compound have been designed. Keeping in view the chemical structure of the lead compound, i.e., the hydrophobic features, HBD's, HBA's, the analogues have been developed. The analogues of the lead have been shown in Fig. 4. The analogues have been developed by the introduction or elimination of various groups. In the present study new analogues were formed by clammenson reduction, acetylene formation and ester formation. The first analogue oflead has been made by reducing ketones into hydrocarbon so in this way hydrophobic character has been increased.In acetylene formation double bonded hydrogen has been replaced by triple bond in order to increase the hydrophobicity of the compound. In case of third analogue $\mathrm{OH}$ group has been replaced by an ester in order to increase the polarity of the compound.

Docking of the analogues through AutodockVina has been performed in order to get the active conformations of the analogues. The interactions of the active conformations of each of the analogue bound into the active site of the protein have been obtained using VMD. The interactions of the analogues with the protein have been shown in Table 3. On the basis of the interactions the analogues have been ranked for being the active ligands. Analogue 2 amongst the all is most active one so it is given the first rank, while second rank has been given to analogue 1, and third to analogue 3.The hydrogen bonds identified in analogues 1,2 and 3 are 4,7 and 2 in number, respectively, the hydrophobic interactions are 4,2and 0 respectively and the Vander Waal interactions are2, 3and 0 respectively. The binding interactions of the most active analogue have been shown, in its docked conformation in Fig. 5. Analogues like the lead compound showed high number of binding interactions. This suggests that the designed analogues are also active like the lead compound.An active antioxidant acting as tyrosine kinase inhibitor has been identified as a lead compound and three active novel analogues have been made which after their analysis are being proposed for clinical trials along with the lead compound for the treatment of Human Cancers caused by the disruption of tyrosine kinase.
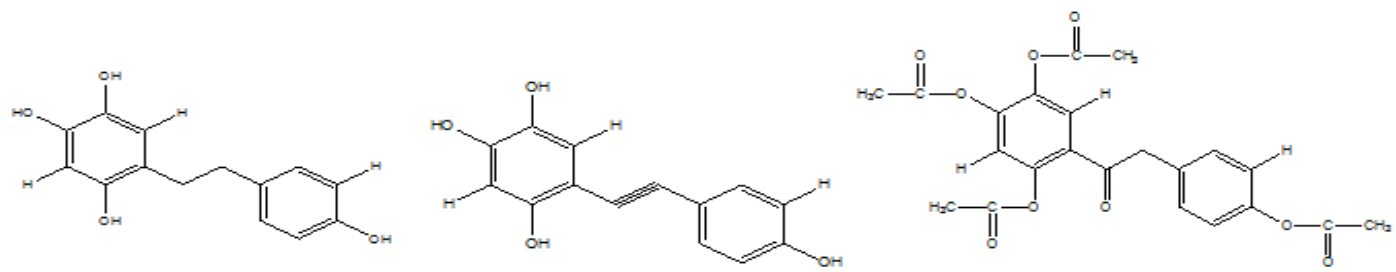

Figure 4. Analogues of lead compound 
Table 3.Binding interactions of analogues of lead compound

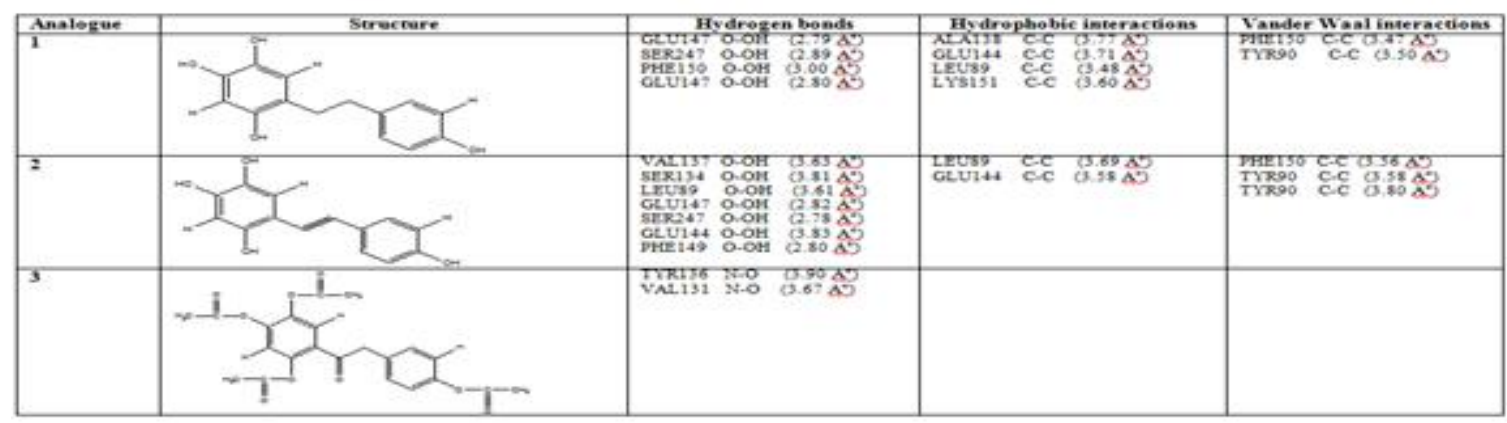

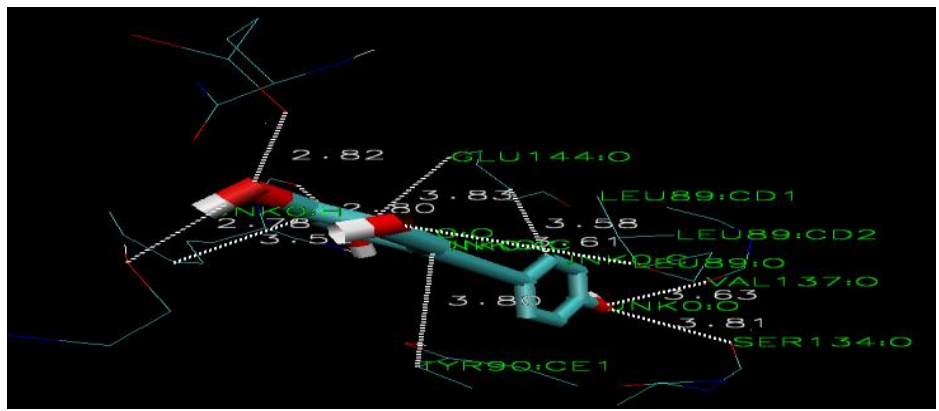

Figure 5.Binding interactions of second analogue with target $2 \mathrm{HCK}$

\section{Conclusion}

The current study was aimed at finding novel antioxidant molecules as tyrosine kinase inhibitors, hence as anticancer inhibitors.In this study various intermolecular interactions between target protein binding site of $2 \mathrm{HCK}$ and different antioxidant compounds were observed. The purpose of this study was to evaluate therapeutic applications of antioxidants in cancer through molecular docking. The study identified an antioxidant Lead compound working as tyrosine kinase inhibitor, hence working as anticancer drug. The active novel analogues of the lead compound were also suggested for their anticancerous activity like the lead compound.

\section{References}

[1] Kang TS, Jo HO, Park WK, Kim JP, Konishi Y, Kong JY, Parka NS and Junga YS, Synthesis and antioxidant activities of 3, 5dialkoxy-4- hydroxycinnamamides, Bioorganic and

[2] Medicinal Chemistry Letters 18, 2008, pp. 1663-1667.

[3] Smietana M, Clayette P, Mialocq P, Vasseur JJ and Joe, A Synthesis of new N-isobutyryl-L-cysteine/MEA conjugates: Evaluation of their free radical-scavenging activities and anti-HIV properties in human macrophages, Bioorganic Chemistry 36, 2008, pp. 133-140.

[4] Cox, Nelson M and David R, Principles of Biochemistry (WH Freeman \& Co,2008).

[5] Lamson DW and Brignall M, Antioxidants and Cancer III:Quercetin, Altern Med Rev 5 (3), 2000, pp.196-208.

[6] Minich DM, Quantum Supplements: A Total Health and Wellness Makeover with Vitamins, Minerals, and Herbs(Conari Press 113, 2010).

[7] K.R. Cousins, "ChemDraw Ultra 9.0. Cambridge soft, 100 Cambridge ParkDrive, Cambridge, MA 02140,” J.Am.Chem.Soc., 127, 2005,pp. 4115-4116.

[8] Chang MW, Ayeni C, Breuer S, Torbett BE, Virtual Screening for HIV Protease Inhibitors: A Comparison of AutoDock 4 and Vina, PLoS ONE 5(8), 2010, el1955.

[9] Humphrey W, Dalke A, Schulten K, VMD: visual molecular dynamics. J Mol Graph 14, 1996, pp. 33-38

[10] $\mathrm{Ng} \mathrm{L}, \mathrm{Ko} \mathrm{HH}$ and Lu TM, Potential antioxidants and tyrosinase inhibitors from synthetic polyphenolicdeoxybenzoins, Journal of Bioorganic and Medicinal Chemistry 17, 2009, pp. 4360-4366.

[11] Calliste CA, Kozlowski D, Duroux JL, Champavier Y, Chulia AJ and Trouillas P, A new antioxidant from wild nutmeg, Journal of Food Chemistry 118, 2009, pp. 489-496.

[12] Kumar V and NagarajaNaik, Synthesis and antioxidant properties of some novel 5H-dibenz[b,f]azepinederivatives in different in vitro model systems, European Journal of Medicinal Chemistry 45, 2010, pp. 2-10.

[13] Kus C, Ayhan G, Ozbey S, Kaynak FB, Kaya M, Coban T and Eke BC, Synthesis and antioxidant properties of novel methyl-1, 3, 4thiadiazol-2-amine and 4-methyl-2H-1, 2, 4-triazole-3(4H)-thione derivatives of benzimidazole class, Journal of Bioorganic \& Medicinal Chemistry 16, 2008, pp. 4294-4303.

[14] Rao YK, Geethangili M, Fang SH and Tzeng YM, Antioxidant and cytotoxic activities of naturally occurring phenolic and related compounds: A comparative study, Journal of Food and Chemical Toxicology 45 (9), 2007, pp. 1770-1776.

[15] Stefek M, Snirc V, Djoubissie PO, Majekova M, Demopoulos V, Rackova L, Bezakova Z, and Wermuth CG, Ganellin, CR, Lindberg $\mathrm{P}$ and Mitscher LA, Glossary of terms used in medicinal chemistry, Elsevier 33, 1998, pp. 385-395. 